\title{
Edge Detection in Ultrasound Images Based on Modified Unsharp and Wavelet transform Filters
}

\author{
Jamila Harbi, Batool Daraam, Waseem M. Ali \\ University of Technology, Iraqi \\ E-mail address: wmamj@yahoo.com
}

\begin{abstract}
Keywords: Unsharp filter; Boost Filter; ultrasound Image; Edge Detection and Extraction; Wavelet Transform; Haar
\end{abstract}

\begin{abstract}
Some image processing applications like segmentation need effective techniques that work as edge detection and extraction, many filters in this field fail to achieve the desired result and consequently the further processing fails, so it is needed sometimes to modify a technique to work in robust and effective way. In ultrasound, although it is a common and real-time non-destructive test methods, but processing and analyzing such images require special filters and modifications to overcome some weakness sides in this field, especially when scanning objects comparable to the acoustic wavelength. In this paper, two modified filters were suggested, first one is two-step unsharp filter, in this techniques the image was enhanced twice the first time the edges that extracted from the original scaled image where added back to the image, and in the second time the same edges were added to the scaled enhanced image. The second technique is summarized as adding back the Low-High and High-Low bands that were extracted previously from the original image by Haar wavelet transform to the image which reinforce its edges.
\end{abstract}

\section{INTRODUCTION}

The traditional unsharp filter (also known as boost filter) is a technique of computing the subtraction between the input image and a blurred (low-pass filtered) version of the input image [OGE], [SOL]. The purpose of this technique is to increase high frequency (fine details) which is interpreted as object edges ${ }^{[\mathrm{OGE}]}$. This operator produces an edge image from the original image using the following methodology:

$$
I_{\text {edge }}(i, j)=I_{\text {original }}(i, j)-I_{\text {smooth }}(i, j)
$$

The smoothed version of the image is typically obtained by filtering the original with low pass filter such as mean or Gaussian filter. Adding the resultant edge image to the original one enhances the edge of the original image as following ${ }^{[\mathrm{SOL}]}$ :

$$
I_{\text {enhanced }}(i, j)=I_{\text {original }}(i, j)+k \times I_{\text {edge }}(i, j)
$$

Here $k$ is the scaling factor, which is an arbitrary value varies between 0.2 to 0.7 preventing the excess of each pixel value over 255 in common uint 8 grayscale images.

Haar is the simplest member of DWT (Discrete Wavelet Transform) family, its procedure is to scan the square image in two steps horizontally and vertically indicating Low-Low, Low-High, High-Low and High-High frequencies in four portions in the resultant image ${ }^{[\mathrm{CHE}]}$. figure 1 shows how DWT indicates the four portions with respect to frequencies in the resultant image. 


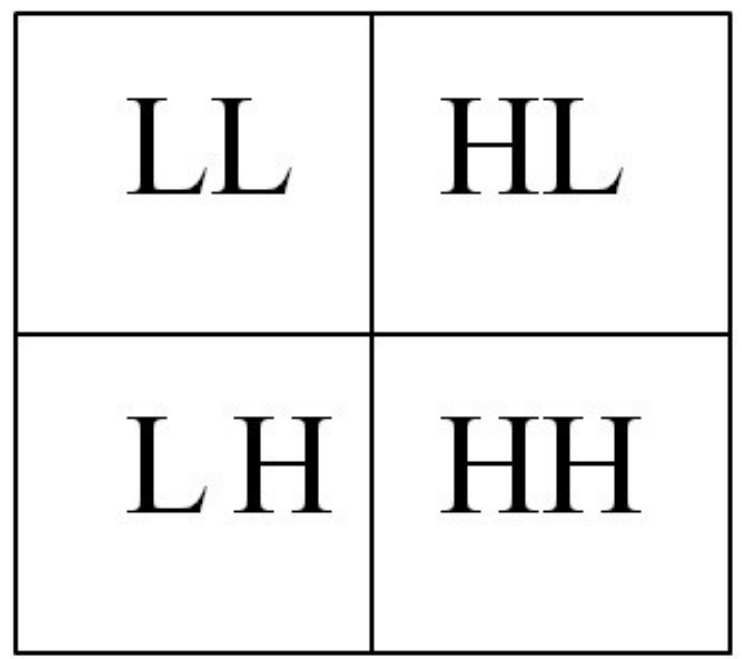

Fig.(1): Discrete wavelet transform

\section{UNSHARP FILTER}

Various filters used for edge detections, taking into consideration that edges (which are high intensity pixel values within the dynamic range of any image) are discontinuities, the first order and second order (Laplacian) derivatives, in addition to sobel and prewitt, play a central role in edge detection and extraction. Also, smoothing filters like mean, median, Gaussian and other similar filters can perform edge detection process rather than noise removal task if the employed to act as unsharp parameter as in eq. 1. Notice that subtracting degraded image with one of the above smoothing filters in eq.1 $\left(I_{\text {smooth }}(i, j)\right)$ from the highly detailed one $\left(I_{\text {original }}(i, j)\right)$, extract effectively the fine details or namely the pixels with high intensity values that mentioned above in this section ${ }^{[\mathrm{OGE}],[\mathrm{SOL}]}$. Adding the extracted edges from eq. 1 to the weighted original image with scaling factor $k$ (eq.2) can enhance the original one by reinforcement edges for further processing like edge-based segmentation as an example ${ }^{[\mathrm{SOL}]}$.

\section{DISCRETE WAVELET TRANSFORM}

Similarly DWT has the effect of edge detection in different applications in image processing as in image segmentation, where edges must define the curves that outline the object in the image ${ }^{[\mathrm{HER}]}$. Adding the edges extracted using DWT to the image may reinforce the image edges.

\section{APPLYING THE UNSHARP MASK}

Figure 2 shows an ultrasound grayscale image, to smooth this image and remove noise, Gaussian filter was chosen with mask size $[5 \times 5]$ and standard deviation $(\sigma=20)$, the result is shown in figure 3. 


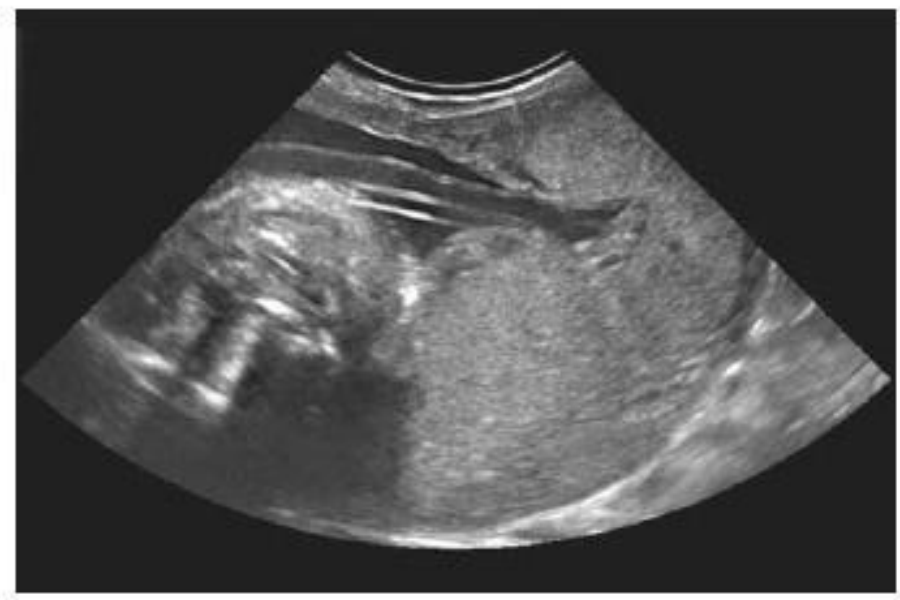

Figure (2.) Original ultrasound image

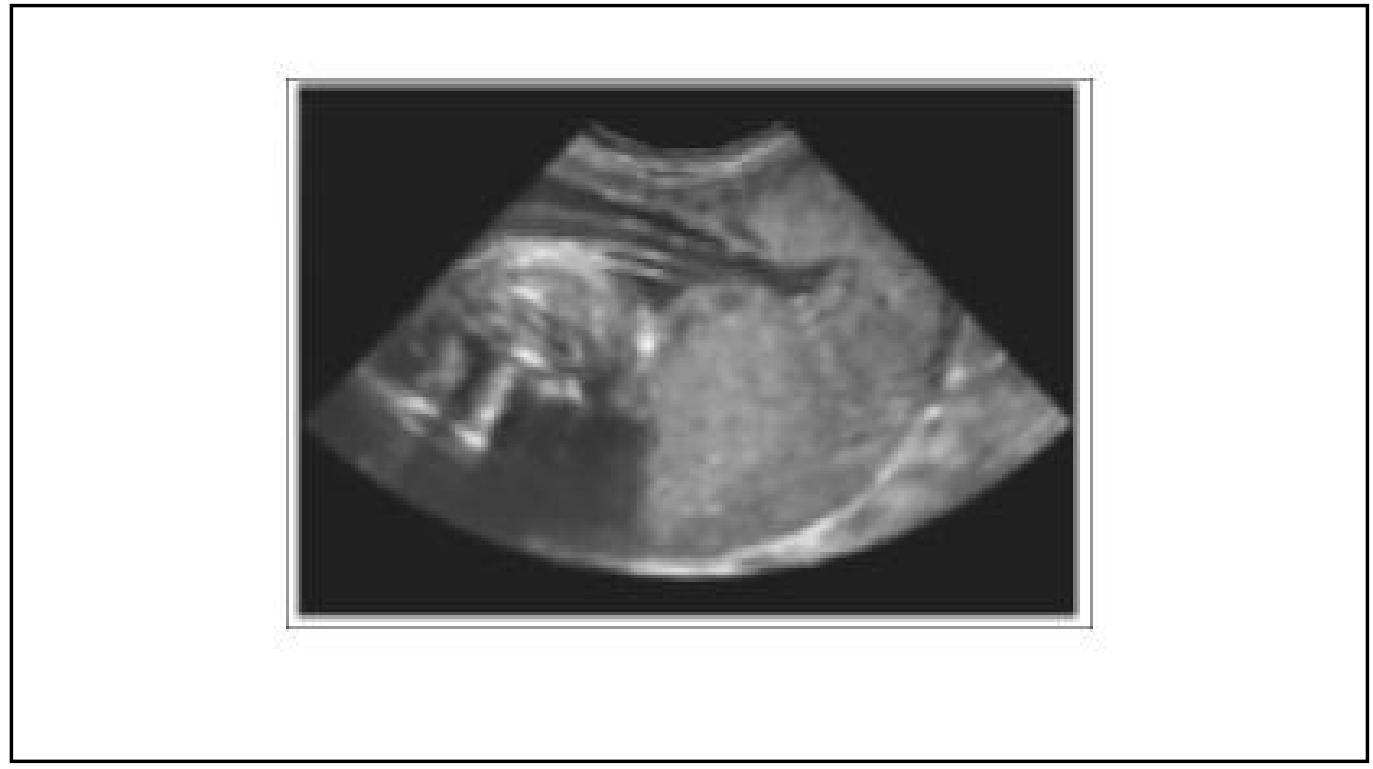

Figure (3). Applying Gaussian filter $[5 \times 5]$ and $\sigma=20$

As we see blurring the image due to usage of Gaussian filter. Now according to eq. 1 , it is suitable to use absolute difference between Fig. (2) and Fig. (3) As follows: 


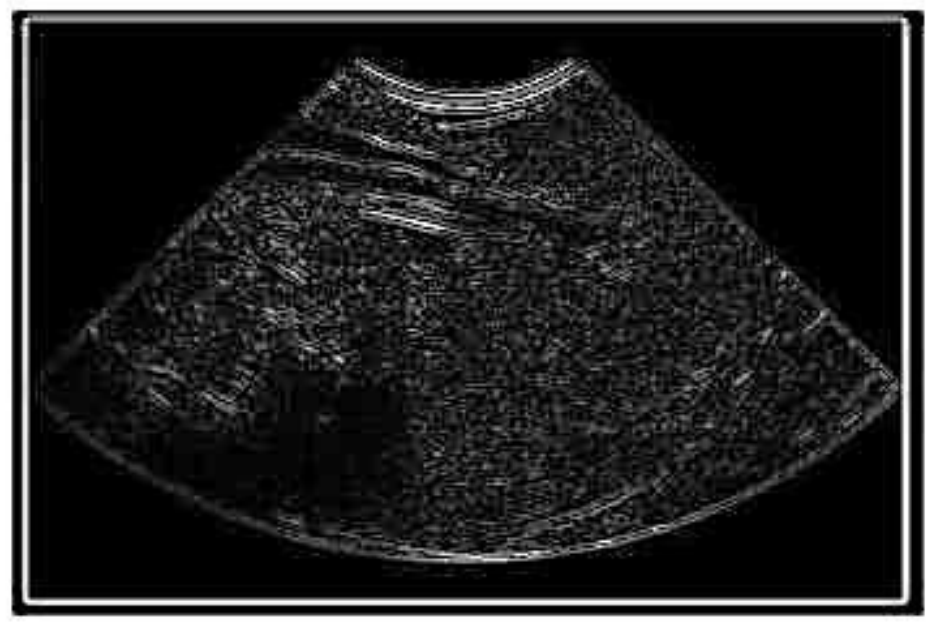

Figure (4) Applying absolute difference between original and filtered images

As shown it is effectively extracted the high intensity pixel values from the original image. Now adding these edges to the original image back with scaling factor mentioned previously $(k=0.7)$ we get the following result:

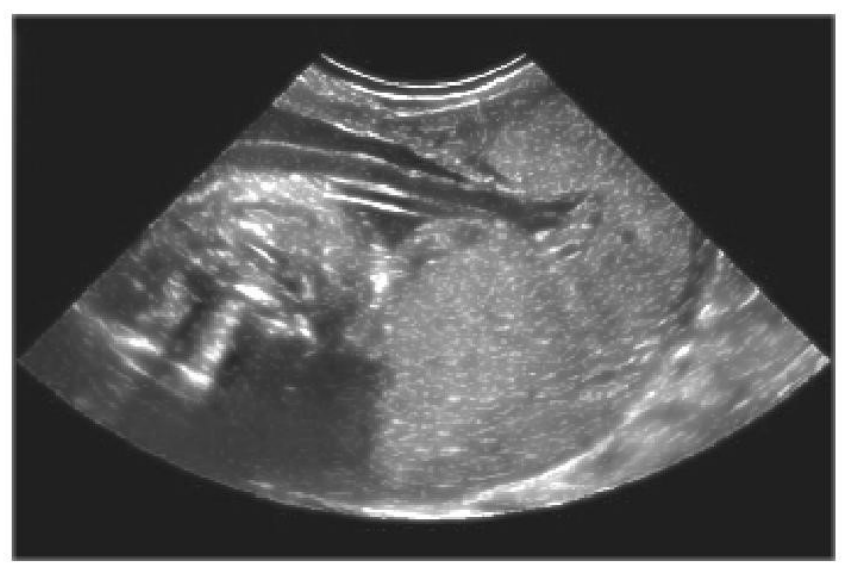

Figure (5) Applying unsharp filter according to eq.2

Supposing a modified unsharp filter which works in two steps of sharpening as in the next equation:

$$
I_{\text {modified }}(i, j)=I_{\text {enhanced }}(i, j)+k \times I_{\text {edge }}(i, j)
$$

This means that the second step performs adding scaled enhanced image of eq.2 to the edges extracted in eq. $1,(k=0.7)$ used. 


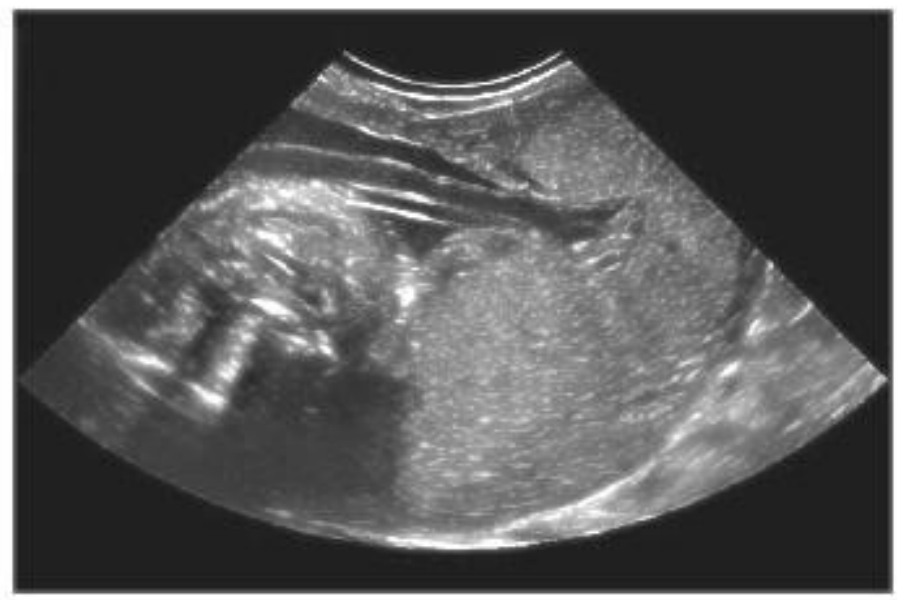

Figure (6) Applying modified unsharp filter according to eq.3

\section{APPLYING WAVELET TRANSFORM:}

In this part the LH and HL portion of Harr wavelet transform were used because these two bands consist of the most edges details of the image scanned horizontally and vertically, LL has now useful edges due to the most details of the image included and conversely the HH has very rare high intensity pixels which generally form no explicit edges, then adding these two bands (LH and HL) to the original image (fig.2) reinforces its edges. 
$\mathrm{HH}$ band of image

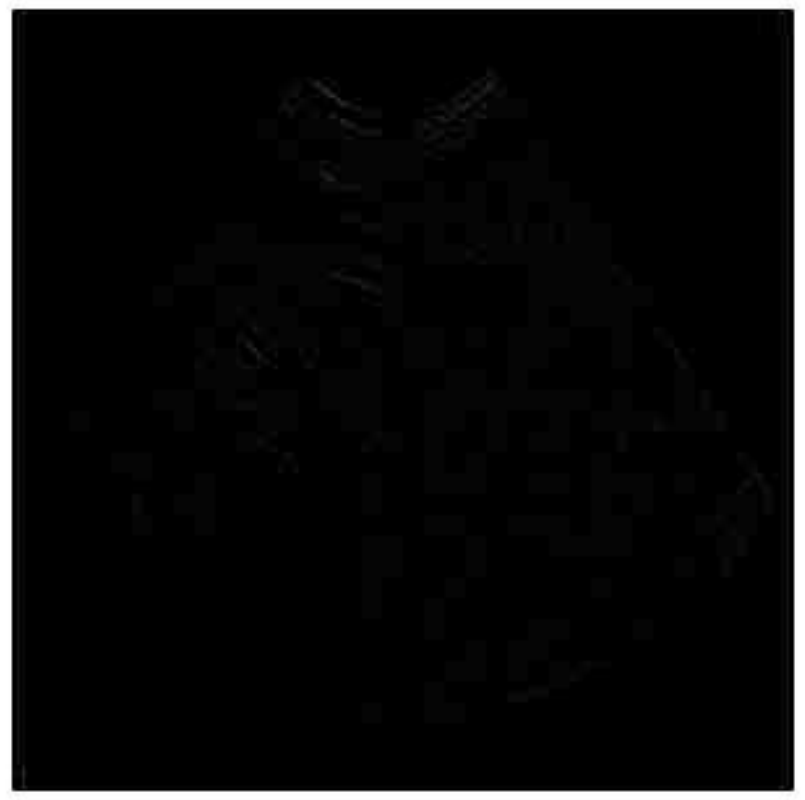

Figure (7) HH band of image shown in fig. 2 using wavelet transform

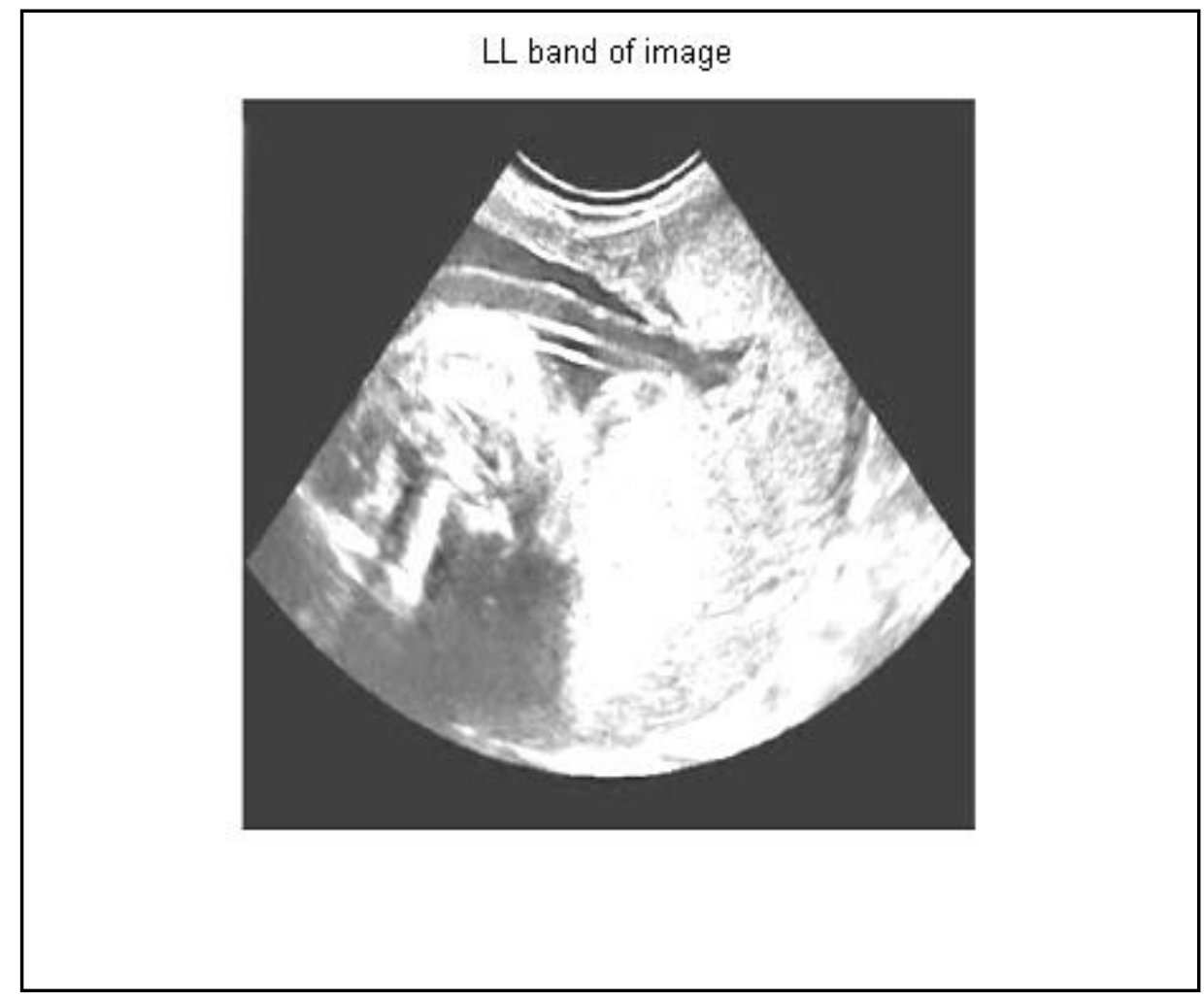

Figure (8) LL band of image shown in fig. 2 using wavelet transform 


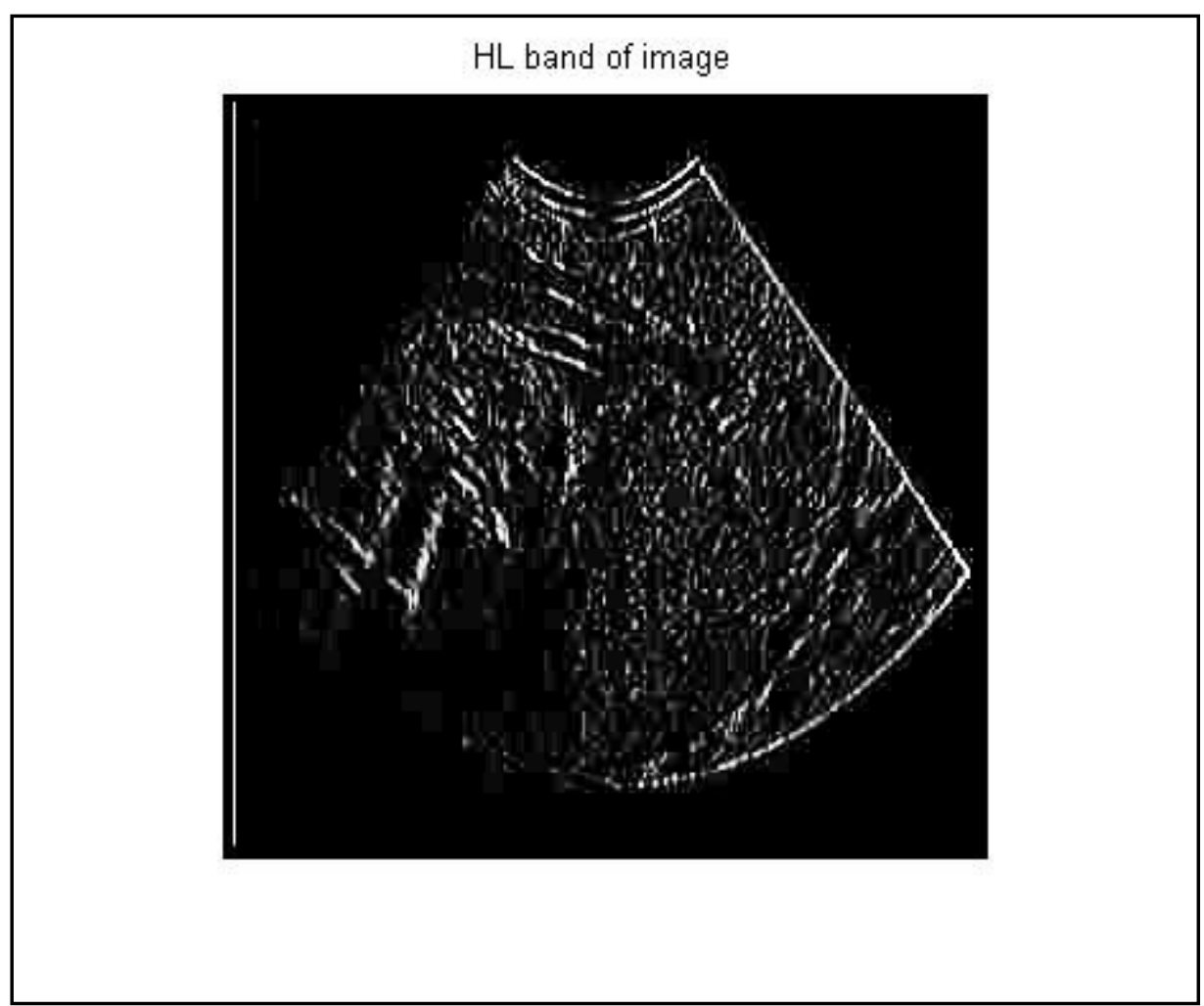

Figure (9) HL band of image shown in fig. 2 using wavelet transform

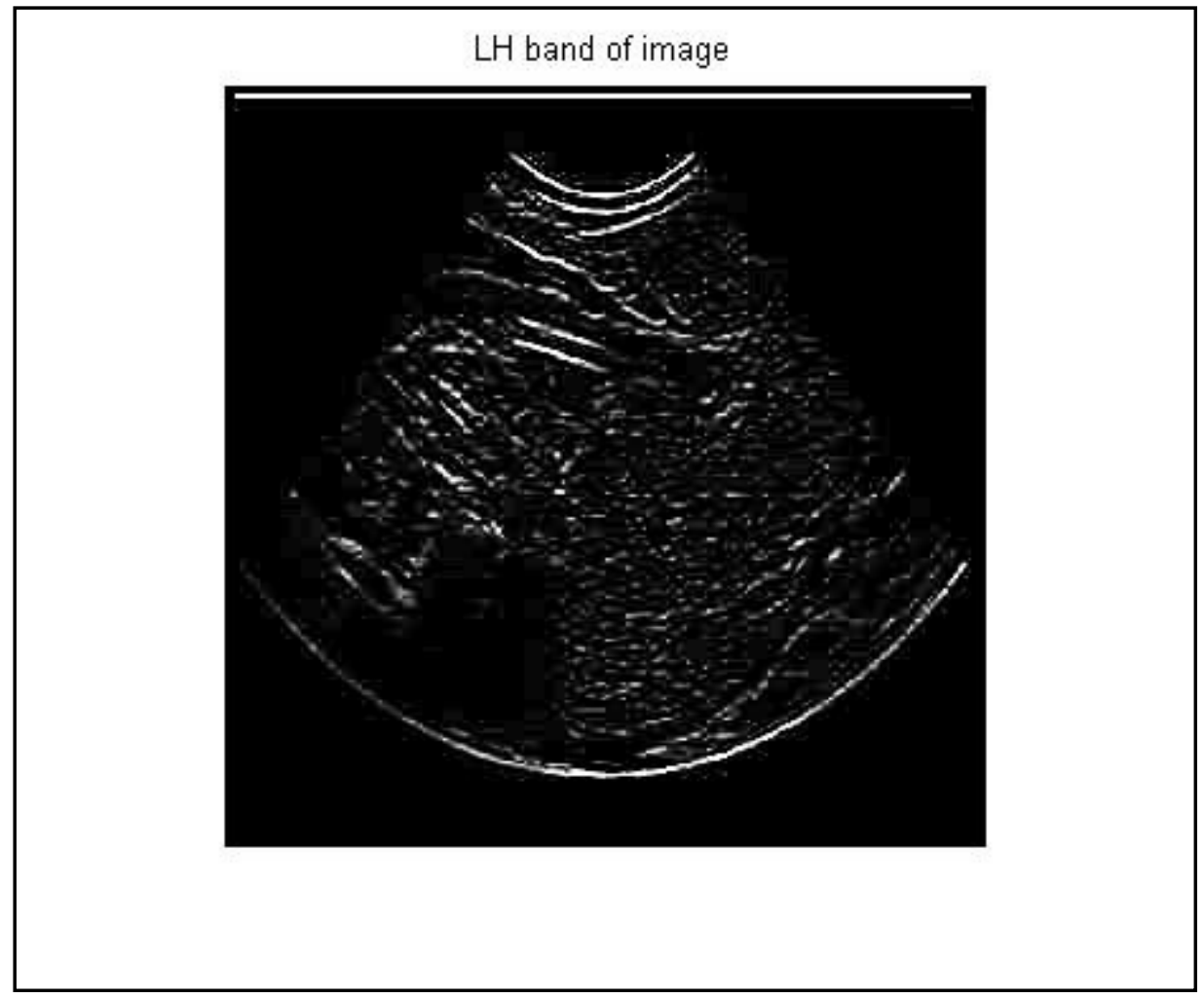

Figure (10) LH band of image shown in fig. 2 using wavelet transform 
Adding the images shown in Fig (9) and Fig (10) to the original image shown in Fig (2) we get the following image:

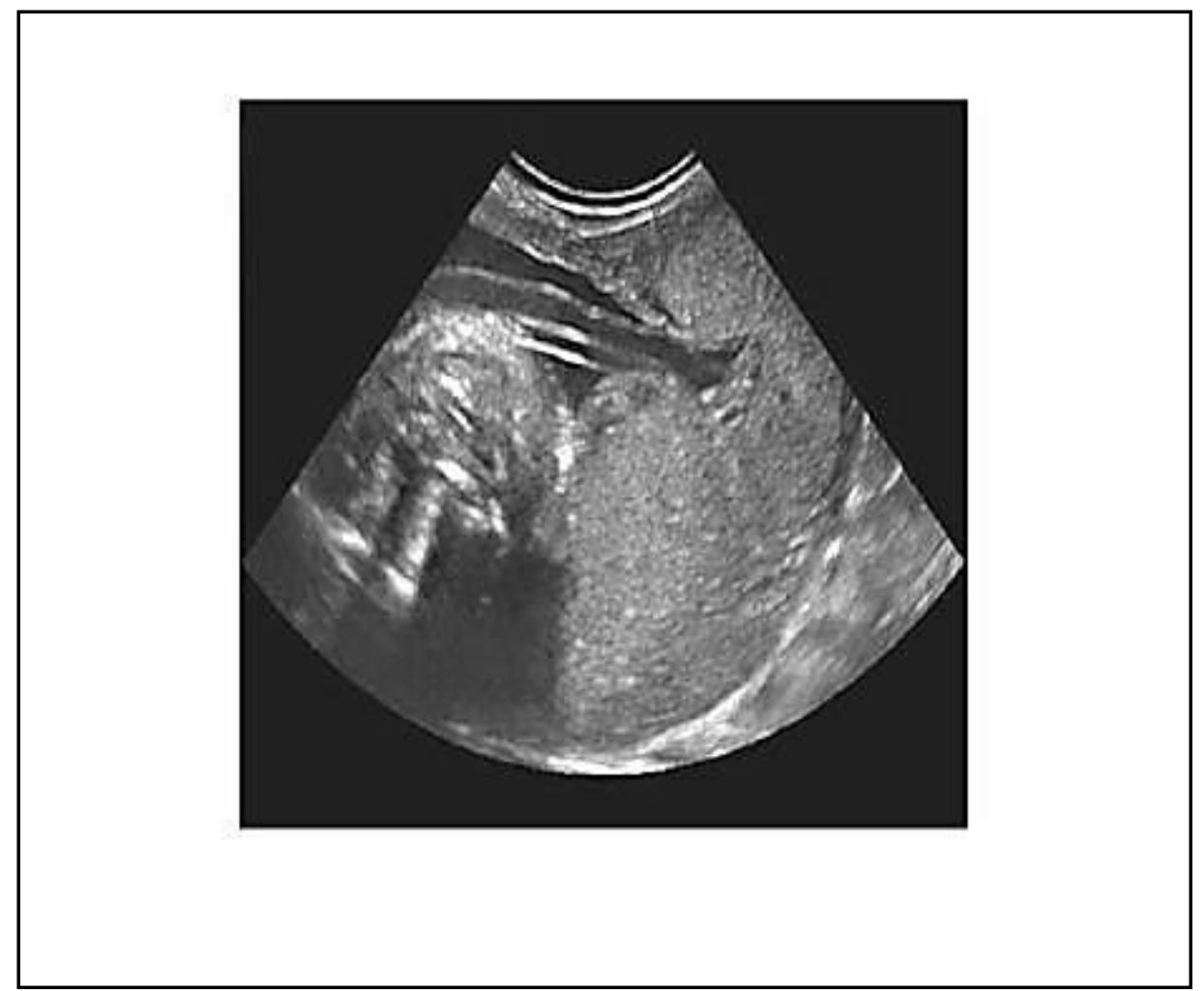

Figure (11) adding LH and HL bands to the original image shown in fig.(2)

\section{RESULTS AND DISCUSSION}

By taking the histogram of each enhanced image by both ways, as shown in figures below, it is clear in figure (13) there is some appearance of peaks that were not exist in figure (12) also shifting the histogram in figure (13) above at the small pixel values means an increment in sharpness and contrast in the filtered image.

Applying Haar Filter shows high contrast in the image (Fig. 11) and as shown in the related histogram (Figm14), where the horizontal axis in any image histogram stands for the pixel values, and the vertical axis for the number of pixels with specific value. 


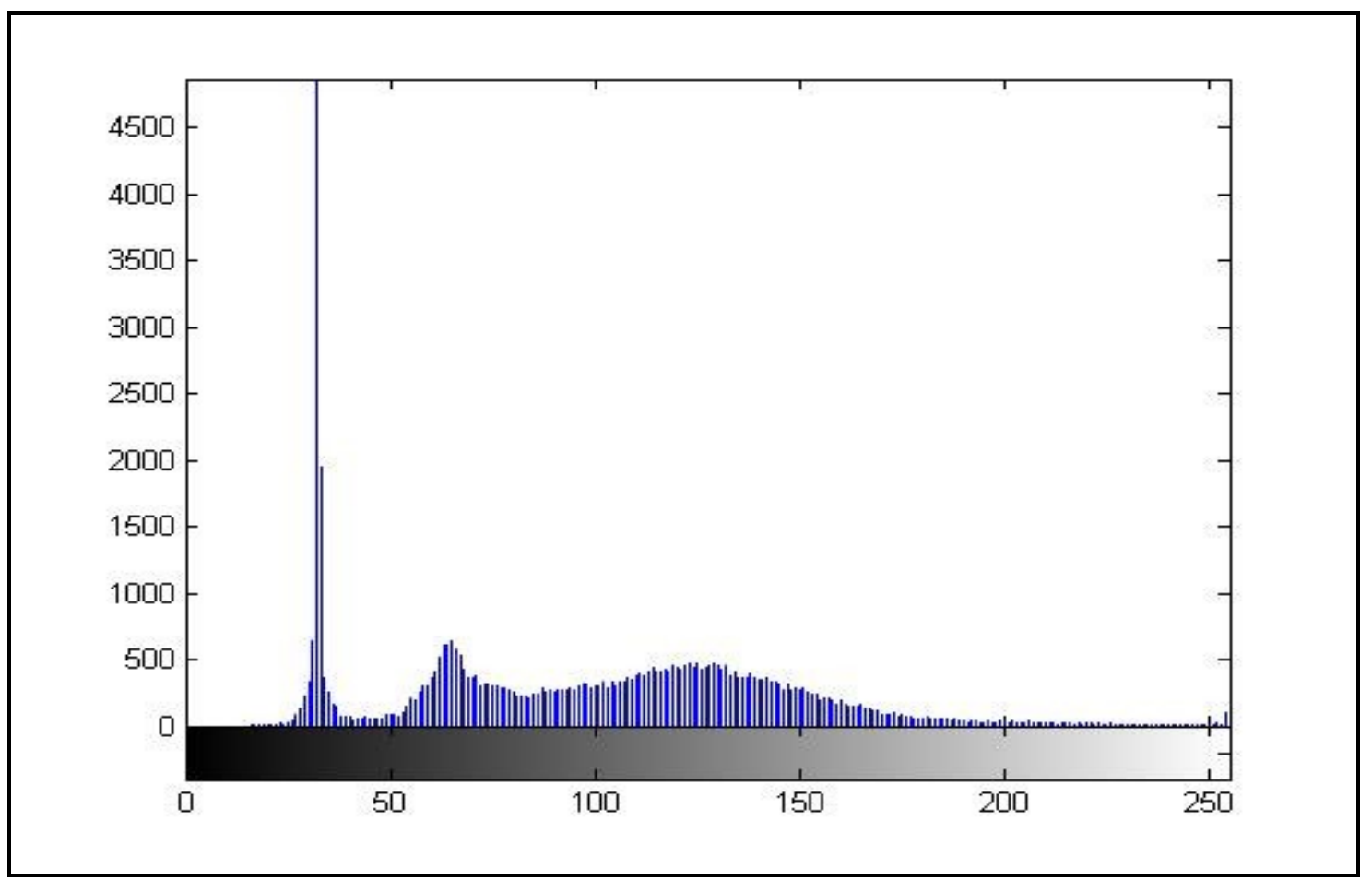

Figure (12) Histogram of original image shown in fig (2)

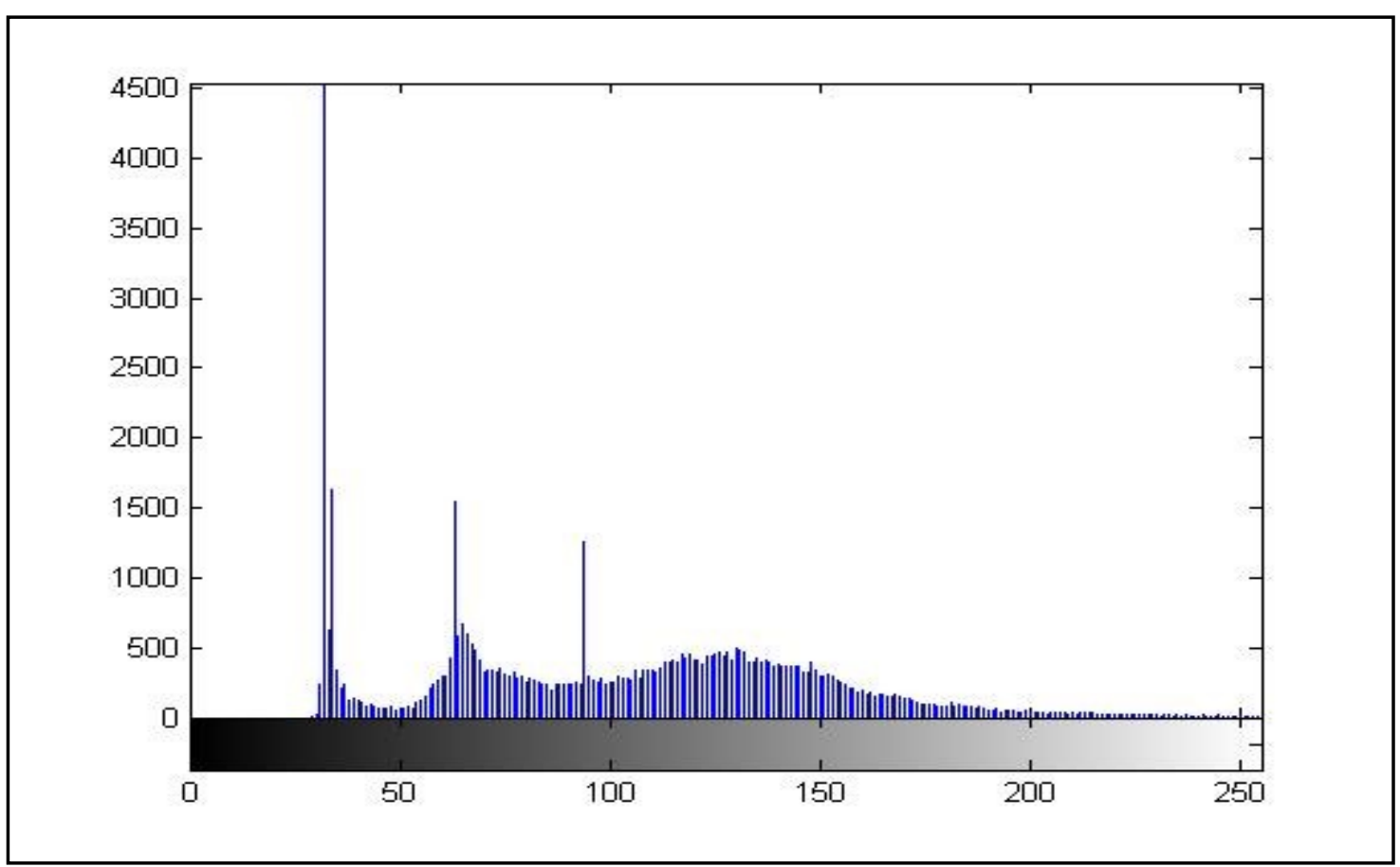

Figure (13) Histogram of the image filtered by modified unsharp filter shown in fig. (6) 


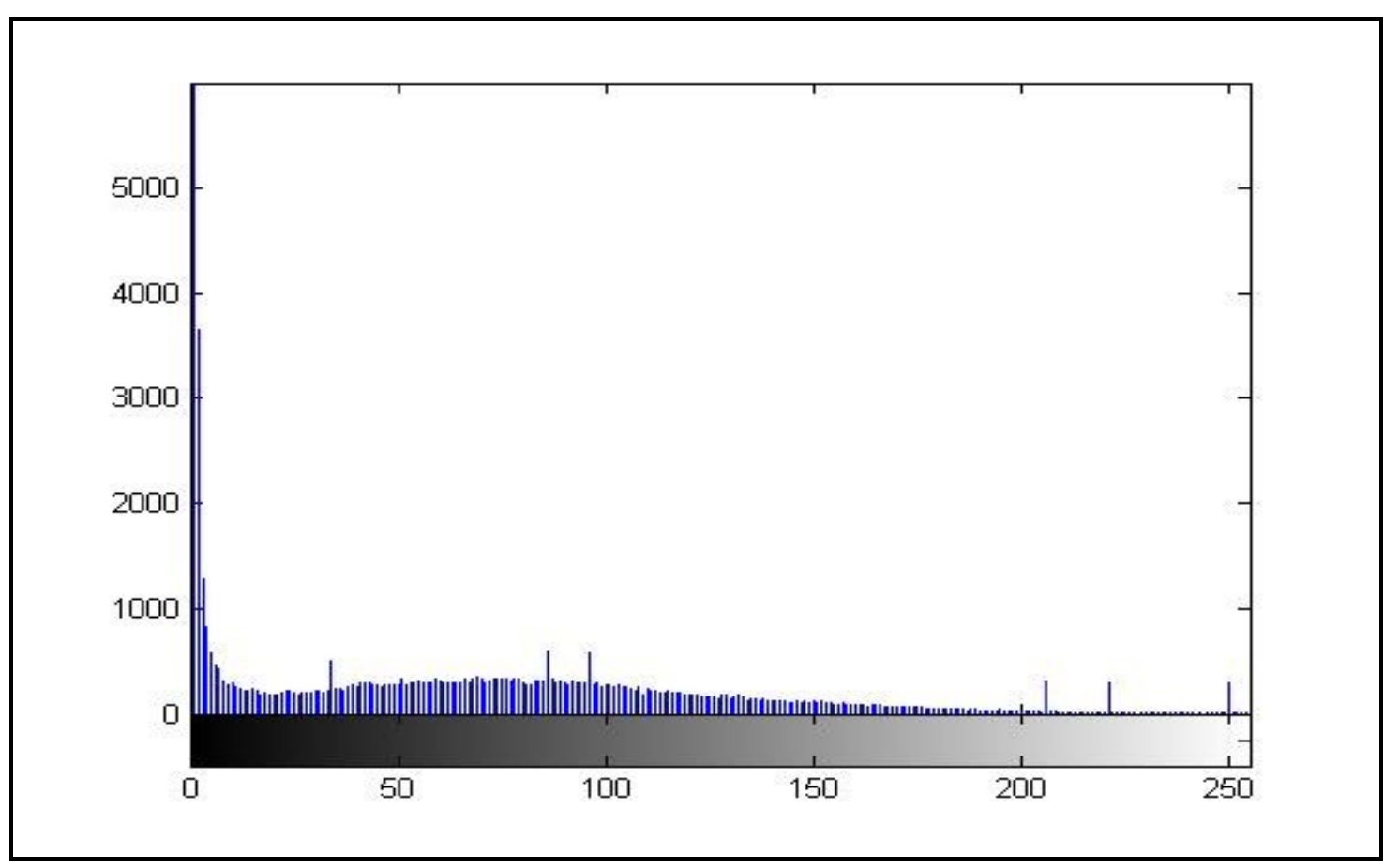

Figure (14) Histogram of the enhanced image by Harr shown in fig.(11)

\section{References}

[1] OGE MARQUES, (2011), "Practical Image And Video Processing Using MATLAB®".

[2] Solomon C., Berckon T.,(2011), "Fundamentals of Digital Image Processing A Practical Approach with Examples in Matlab".

[3] Po-Yueh Chen and Hung-Ju Lin,International Journal of Applied Science and Engineering,2006, "A DWT Based Approach for Image Steganography"

[4] Dušan Heric , Damjan Zazula,Image and Vision Computing 25 (2007) 652-662, SienceDirect, "Combined edge detection using wavelet transform and signal registration". 